\title{
Effects of sensory stimulation on upper limb strength, active joint range of motion and function in chronic stroke virtual reality training
}

\author{
Dong-Hoon Kim ${ }^{a}{ }^{(\oplus)}$, Suk-Min Lee ${ }^{\mathrm{b}(1)}$ \\ ${ }^{a}$ Department of Physical Therapy, Gimcheon University, Gimcheon, Republic of Korea \\ ${ }^{b}$ Department of Physical Therapy, College of Health Science and Social Welfare, Sahmyook University, Seoul, Republic of Korea
}

Objective: This study aimed to investigate the upper limb strength, active joint range of motion (AROM), and upper limb function in persons with chronic stroke using virtual reality training in combination with upper limb sensory stimulation.

Design: Two-group pretest-posttest design.

Methods: 20 subjects were divided into two groups of 10, the sensory motor stimulation and virtual reality training (SMVR) and virtual reality training (VR) groups. The training was conducted for 30 minutes per session, three times a week for 8 weeks. The participants' upper limb strength was measured via the hand-held dynamometer, joint angle AROM was measured via dual inclinometer, function was measured using the Jebson-Taylor hand function test and the manual function test.

Results: Significant differences were observed in all groups before and after the training for upper extremity strength, AROM, and function $(p<0.05)$. Between the two groups, the SMVR group showed significant improvement in muscle strength, AROM, and Jebsen-Taylor hand function test scores compared with the VR groups $(p<0.05)$.

Conclusions: In this study, we confirmed that sensory stimulation and VR had positive effects on upper extremity strength, AROM, and function of persons with chronic stroke. The results suggest that in the future, VR in combination with sensory stimulation of the upper limb is likely to become an effective method (a rehabilitation training program) to improve the upper limb function of persons with chronic stroke.

Key Words: Function, Range of motion, Sensory, Stroke, Virtual reality

\section{Introduction}

Stroke is a state in which the blood vessels that provide nutrients to the brain are ruptured, causing blockage and subsequent damage to the brain (World Health Organization, 2016). In the United States, stroke is the fifth leading cause of death, and approximately 800,000 cases are reported each year [1].

Stroke is the main cause for direct loss of function and death, and since most survivors need long-term rehabilitation treatment due to various consequences, it is a condition that causes heavy burden to the family and society as well as the patients themselves [2].

Normal upper body function is a basic necessity for performing delicate movements, such as eating, putting on clothes, or writing, and is a very important element in daily life. Moreover, upper extremities use forms a considerable part of the human activities performed at work [3]. Upper arm dysfunction due to stroke is primarily accompanied by motor and sensory disturbances owing to brain damage, and secondarily accompanied by limitations in various activities of daily living (ADL) [4]. Over 50\% patients with hemiplegia do not recover function on the affected side due to upper extremity paralysis, which disrupts daily life activity

Received: 16 June, 2020 Revised: 15 July, 2020 Accepted: 9 August, 2020

Corresponding author: Suk-Min Lee (ORCID https://orcid.org/0000-0002-6062-956X)

Department of Physical Therapy, College of Health Science and Social Welfare, Sahmyook University, 815 Hwarang-ro, Nowon-gu, Seoul 01795, Republic of Korea Tel: 82-2-3399-1632 Fax: 82-2-3399-1639 E-mail: leesm@syu.ac.kr

(c) This is an Open-Access article distributed under the terms of the Creative Commons Attribution Non-Commercial License (http://creativecommons.org/licenses/ by-nc/4.0) which permits unrestricted non-commercial use, distribution, and reproduction in any medium, provided the original work is properly cited.

Copyright $@ 2020$ Korean Academy of Physical Therapy Rehabilitation Science 
performance. In addition, the impaired functions persist in the upper extremities even after treatment [5].

Sensorimotor training is an exercise controlled by the integrated information of the brain by maximizing the proprioceptive stimulation of the central nervous system through centripetal information stimulation [3]. Furthermore, the methods of physical therapy that emphasize sensory stimulation have recently gained importance in rehabilitation strategies, and were reported to be significant in maximizing neuroplasticity and stimulating the above-mentioned sensory systems for patients with stroke in various ways [6].

Recently, several therapeutic techniques have been suggested to promote upper limb recovery in individuals affected by stroke, including robotic therapy [7], virtual reality training (VR) [8], sensory stimulation therapy [9], and imagery training [10]. Out of these therapeutic methods, training interventions for the motor function recuperation utilizing various sensory data, such as visual, tactile, auditory and proprioception, can improve the efficacy of rehabilitation exercises [11].

In particular, when virtual reality rehabilitation was applied using appropriate levels of challenge, the effects on upper limb function and activities of daily living (ADL) performance were beneficial for those affected by stroke [12]. VR creates a simulated environment mimicking actual situations through which the patient can acquire various experiences. As a result of the various interactions as well as internal and external feedback provided through the VR screen, the VR therapy can be used as rehabilitation training in various areas, such as exercise and cognitive rehabilitation [13,14]. Consequently, the robot intervention method based on VR has become more familiar in recent times. The VR training arouses interest and fun, and increases motivation in performing tasks [15]. It is effective for enhancing cognitive and visual perceptual skills as well as the functions and muscle strength of the upper extremities [16]. Individuals with stroke often complain of problems in processing perception and recognition; in a related clinical study, when VR was applied to patients with spatial orientation problems, their geographical orientation processing ability improved [17].

However, a insufficient amount of attention has been directed towards to the somatosensory function rehabilitation training for individuals with chronic hemiparetic stroke in particular [18]. Also, there are very few studies on the use of virtual reality exercises for upper extremity treatment in chronic stroke survivors. Specifically, studies on the ability to concentrate, the effect of the strength, AROM, and the upper extremity function as well as those involving the lack of sensory motor stimulation in stroke patients are very insufficient. Therefore, the purpose of this study was to examine upper extremity strength, AROM, and function using virtual reality training combined with sensory stimulation in patients with chronic stroke. The findings in this study may provide reference information to establishing interventions for the enhancement of upper extremity function and improvement of ADL performance in those affected by stroke.

\section{Methods}

\section{Participants and procedure}

This study was a two-group pretest-posttest design. Twenty patients who had met the selection criteria from 2 hospitals located in Korea agreed to participate in this study. The inclusion criteria were follows: individuals who were diagnosed with stroke at least 6 months ago with impairments in the affected upper extremity, those without upper limb orthopedic injuries, those without cognitive deficits (Korean version of the Mini-Mental State Examination $\geq 24$ points), those with a manual muscle test (MMT) grade of two or higher of the affected upper extremity, and those with at least $20^{\circ}$ of wrist extension and at least $10^{\circ}$ of extension of the metacarpophalangeal and carpal joints of the affected side, and those who agreed to volunteer for the study. Exclusion criteria were those affected with unilateral neglect, hemianopia, or impaired auditory and visual senses.

Patients received sufficient explanation in writing on the research purpose, content, methods, and confidentiality as well as adequate explanation on the possibility of withdrawing whenever they desired before the study proceeded. The study adhered to the Helsinki Declaration principles and received approval from the Sahmyook University Institutional Review Board (IRB No. 2-1040781-A-N-012020013HR).

20 patients met the selection criteria and were randomly assigned to sensory motor stimulation and virtual reality training (SMVR) and virtual reality (VR) $(n=10$, respectively) through a computer program. The training was implemented for 8 weeks, and evaluations were conducted 8 weeks pre-post training, respectively.

\section{Intervention}

The study was organized from January to March 2020 including a total of 20 subjects who were divided into two groups of 10, which were the SMVR and VR groups. The in- 


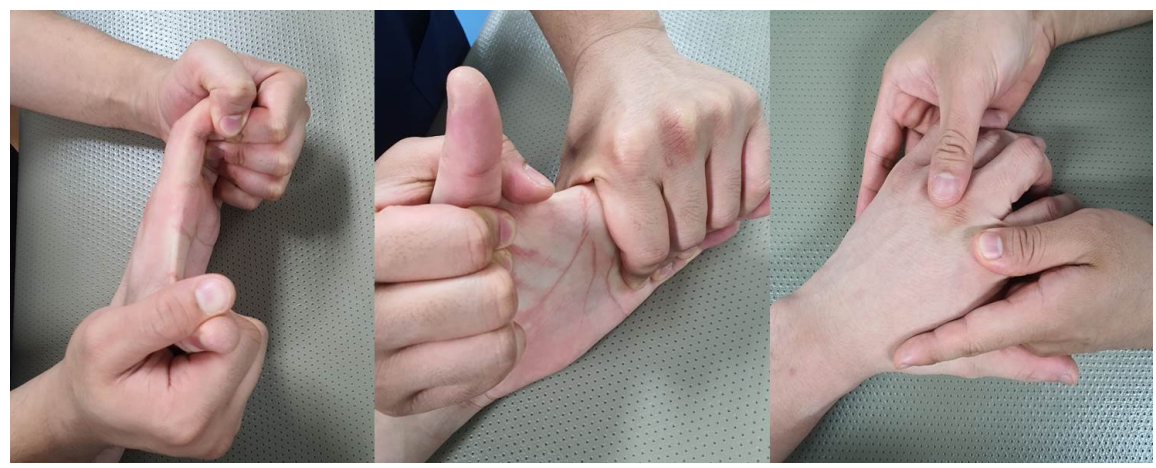

Figure 1. Sensory motor stimulation exercise.

tervention was conducted for 30 minutes per session, 3 times a week for 8 weeks.

In the SMVR group, sensory-motor stimulation was implemented for 30 minutes, 3 times for 8 weeks, and VR was implemented for 30 minutes. In the VR group, VR was implemented for 30 minutes, 3 times for 8 weeks, and conservative therapy was administered for 30 minutes.

The sensory motor stimulation training applied in this study was modified from the sensory motor stimulation program used in a study by de Diego et al. [7]. The patient sat straight on the chair and placed the affected hand on the desk. This stimulation training consisted of 5, 5, 10, and 10 min of hand therapy, sensory input, sensory therapy, and functional motion, respectively. Hand therapy consisted of metacarpophalangeal joint pressure, thenar/hypothenar muscle mobilization, and interosseous muscle mobilization. For the sensory input, each corresponding part of the hand was stimulated using a brush. Items were selected by stretching the arm toward objects at various angles, moving a bag, grasping balls of various sizes, and opening a door for assessment of functional motion (Figure 1).

VR was performed using the Smart Glove (Neofect, Yongin, Korea), which is a biofeedback machine used for upper extremity rehabilitation of persons with stroke and consists of a machine attached with glove-shaped sensors and a software application. Smart Glove games include various upper extremity movements, which include forearm supination/pronation, wrist flexion/extension, wrist joint radial/ulnar deviation in finger flexion/extension, and additional complex movements. The person who wears the smart glove is expected to perform various tasks, including catching balls, fishing, squeezing an orange, cooking, pouring wine, cleaning the floor, turning pages of a book, and painting (Figure 2).

Conservative therapy-neurophysiological therapy, such

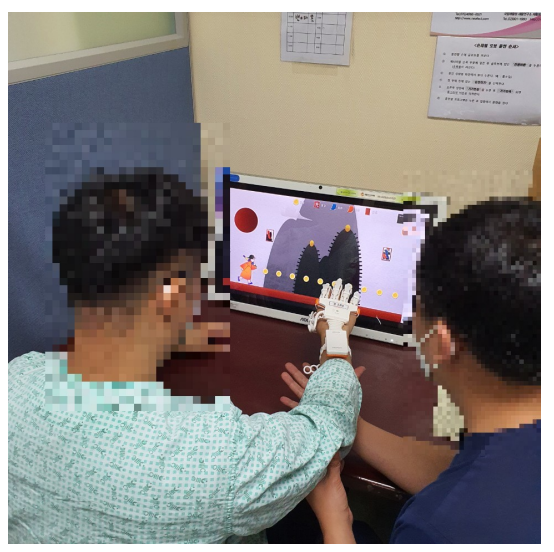

Figure 2. Virtual reality training.

as neurodevelopmental physical therapy, was applied to the paralyzed sidealong with stretching exercises.

\section{Outcome measures}

A hand-held dynamometer (Commander Echo Muscle Tester; JTECH Medical, Midvale, UT, USA, 2004) was used to assess changes in the active muscular strength of the subject's upper extremity. When measuring the flexors and extensors of the elbow and wrist joints, both arms of the participations were placed on the desk, with the hand-held dynamometer sensor placed on the metacarpal of the hand so as to measure muscular strength as the wrist was flexed and extended. The intra-rater reliability was $\mathrm{r}=0.90-0.96$; and inter-rater reliability was $\mathrm{r}=0.76-0.97$ [19].

A dual inclinometer (Dualer IQ Inclinometer, JtechMedical, USA, 2005) was used to assess the AROM of bilateral elbow and wrist flexion and extension. When measuring the ROM for elbow and wrist joint flexion and extension, one sensor was attached to the forearm, and another sensor was attached to the upper arm using velcro. The intraclass correlation coefficient of the electronic inclinometer 
was $\geq 0.95$ [19].

The Jebsen-Taylor hand function test was used to assess with the patient sitting comfortably in a chair that was in front of a desk placed opposite to the tester in a bright place without visual hindrance. During the assessment, 7 items were examined using a standardized stopwatch, and the non-dominant hand was measured first. The patient practiced 10 times for each test item before the assessment, and the mean values obtained from the 3 measurements were used for data analysis [20]. The internal consistency of this instrument for evaluation was 0.98 , and the inter-rater reliability was $\mathrm{r}=0.90-0.99$ [21].

The Manual Function Test was used, which consists of upper limb movement (four items), grip (two items), and finger manipulation (two items), with 1 point scored for each performed test and 0 point for each failed test, with a perfect score of 32. The test-retest reliability and inter-tester reliability were set at $\mathrm{r}=0.095$ [22].

\section{Data and statistical analysis}

The IBM SPSS Statistics for Windows, Version 20.0 (IBM Co., Armonk, NY, USA) was used for all statistical analyses in this study. The Komogorov-Smirnov test was used to assess normal distribution. The gender, stroke type, and paralyzed side were examined by the chi-square test, and the homogeneity of variables between the 2 groups before intervention was tested by the independent sample $t$-test. The paired t-test was performed to determine the differences pre and post treatment within the group, and the independent sample t-test was performed to contrast the differences between the groups. All statistical significance degree were set at $\alpha=0.05$.

\section{Results}

The general characteristics of participants are shown in Table 1. Significant differences $(p<0.05)$ were confirmed in all groups before and after the training for upper limb strength, AROM, and function. Between the 2 groups, there were significant improvement in muscle strength, AROM, and Jebsen-Taylor hand function test scores in the SMVR group compared with the VR group $(p<0.05)$ (Table 2$)$.

\section{Discussion}

This study aimed to examine the impacts of sensory motor stimulation and VR on a chronic stroke patient's upper limb
Table 1. General characteristics of subjects $(\mathrm{N}=20)$

\begin{tabular}{lrrr}
\hline \multicolumn{1}{c}{ Group } & \multicolumn{1}{c}{$\begin{array}{c}\text { SMVR } \\
(\mathrm{n}=10)\end{array}$} & \multicolumn{1}{c}{$\begin{array}{c}\text { VR } \\
(\mathrm{n}=10)\end{array}$} & \multicolumn{1}{c}{$\chi^{2} / \mathrm{t}(p)$} \\
\hline Age $(\mathrm{y})$ & $58.8(8.88)$ & $55.9(6.49)$ & $1.353(0.415)$ \\
Height $(\mathrm{cm})$ & $164.2(6.05)$ & $163.5(4.03)$ & $1.296(0.764)$ \\
Weight $(\mathrm{kg})$ & $59.4(7.73)$ & $60.5(5.42)$ & $2.114(0.717)$ \\
MMSE-K (score) & $27.1(1.66)$ & $28.1(1.29)$ & $0.2(0.150)$ \\
Sex & & & \\
$\quad$ Male & $4(40.0)$ & $5(50.0)$ & $0.202(1.000)$ \\
$\quad$ Female & $6(60.0)$ & $5(50.0)$ & \\
Diagnosis & & & \\
$\quad$ Infarction & $5(50.0)$ & $7(70.0)$ & $0.833(0.650)$ \\
$\quad$ Hemorrhage & $5(50.0)$ & $3(30.0)$ & \\
Affected side & & & \\
$\quad$ Left & $3(30.0)$ & $5(50.0)$ & $0.833(0.650)$ \\
$\quad$ Right & $7(70.0)$ & $5(50.0)$ & \\
\hline
\end{tabular}

Values are presented as mean (SD) or $\mathrm{n}(\%)$.

SMVR: sensory motor and virtual reality training, VR: virtual reality training, MMSE-K: mini-mental state examination-Korea version.

muscular strength, AROM, and upper extremity function. Sensory motor training and VR were conducted for 8 weeks, and the upper limb muscular strength, AROM and upper extremity function results were analyzed.

Smania et al. [18] conducted somatosensory training for patients with stroke with sensory damage and reported a recovery of senses and improvement of their perception ability with hand contact recognition response [23]. VR-based task training has recently been reported to be very effective in the rehabilitation of patients with stroke with environmental or physical limitations performing various physical or leisure activities [24].

Increased upper extremity strength was the greatest in the SMVR group. This was consistent with the result of improved muscular strength of the finger extensors and wrist joint as a variable of the upper limb function of the individual with stroke, as presented in the preceding studies of Kim and Lee [25]. In addition, it was consistent with the findings of Powell et al. [26], who reported the significance of the changes in muscular strength, applying electrostimulation to the wrist joint extensor, as well as the findings of Yun et al. [27], who stated that mirror therapy in parallel with functional electric stimulation had a significant effect on the change in finger extensor muscular strength of individuals with stroke. We believe that the improvements in muscular strength resulting from the upper limb interventions using sensory-motor stimulation and VR are paralleled to this study. The direct sensory receptor stimulation in 
Table 2. Comparison of upper limb strength, AROM, and function between the two groups

$(\mathrm{N}=20)$

\begin{tabular}{|c|c|c|c|c|c|}
\hline \multirow{2}{*}{ Variable } & \multicolumn{2}{|c|}{ SMVR $(n=10)$} & \multicolumn{2}{|c|}{$\operatorname{VR}(n=10)$} & \multirow{2}{*}{$\mathrm{t}(p)$} \\
\hline & Pre-test & Post-test & Pre-test & Post-test & \\
\hline EFMT (kg) & $1.71(0.27)$ & $2.23(0.41)^{*}$ & $1.62(0.42)$ & $1.87(0.46)^{*}$ & $2.133(0.047)^{\mathrm{a}}$ \\
\hline EEMT (kg) & $1.76(0.30)$ & $2.17(0.43)^{*}$ & $1.71(0.42)$ & $1.91(0.42)^{*}$ & $3.132(0.009)^{\mathrm{a}}$ \\
\hline WFMT (kg) & $0.91(0.35)$ & $1.27(0.39)^{*}$ & $0.99(0.27)$ & $1.27(0.29)^{*}$ & $2.206(0.041)^{\mathrm{a}}$ \\
\hline WEMT (kg) & $0.81(0.42)$ & $1.10(0.50)^{*}$ & $0.98(0.27)$ & $1.19(0.26)^{*}$ & $1.385(0.183)$ \\
\hline EFAROM $\left(^{\circ}\right)$ & $106.70(5.76)$ & $114.00(7.00)^{*}$ & $105.10(5.26)$ & $108.50(5.50)^{*}$ & $3.239(0.005)^{\mathrm{a}}$ \\
\hline EEAROM $\left(^{\circ}\right)$ & $-32.50(9.88)$ & $-28.00(8.99)^{*}$ & $-30.80(6.37)$ & $-27.730(5.76)^{*}$ & $2.105(0.05)$ \\
\hline WFAROM $\left(^{\circ}\right)$ & $23.70(3.77)$ & $28.60(4.86)^{*}$ & $24.00(4.71)$ & $26.90(5.86)^{*}$ & $2.636(0.017)^{\mathrm{a}}$ \\
\hline WEAROM $\left(^{\circ}\right)$ & $21.20(3.29)$ & $27.30(3.23)^{*}$ & $21.30(4.57)$ & $24.40(4.97)^{*}$ & $3.744(0.001)^{\mathrm{a}}$ \\
\hline JTT (score) & $13.80(2.66)$ & $17.70(3.09)^{*}$ & $12.70(3.62)$ & $15.10(4.09)^{*}$ & $2.330(0.032)^{\mathrm{a}}$ \\
\hline MFT (score) & $13.40(1.90)$ & $17.20(2.20)^{*}$ & $12.90(3.28)$ & $15.90(3.87)^{*}$ & $1.395(0.180)$ \\
\hline
\end{tabular}

Values are presented as mean (SD).

SMVR: sensory motor and virtual reality training, VR: virtual reality training, EFMT: elbow flexion muscle test, EEMT: elbow extension muscle test, WFMT: wrist flexion muscle test, WEMT: wrist extension muscle test, EFAROM: Elbow flexion active range of motion, EEAROM: Elbow extension active range of motion, WFAROM: wrist flexion active range of motion, WEAROM: wrist extension active range of motion, JTT: Jebsen-Taylor hand function test, MFT: manual function test.

${ }^{a}$ Significant difference compared with VR group $(p<0.05)$.

*Significant difference compared with pre-test $(p<0.05)$.

the skin and muscles in sensory-motor training had a direct impact on the unique senses, which led to the improvements of the elbow joint flexor/extensor and wrist joint flexor/extensor strength.

Regarding AROM, the SMVR group showed greater improvement than the VR group. This result was similar to that of a study conducted by Lee et al. [28] that implemented asymmetrical upper limb training using a VR device on stroke patients and reported that there were significant differences in the ROM of wrist flexion, extension, radial deviation, and ulnar deviation. Moreover, there were significant differences in wrist flexion and extension in the control group, which was consistent with the result of this study. Subramanian and Levin. [29] divided 32 patients with chronic stroke into a training group with VR and a training group in the actual environment and intervened in them and reported that there were significant increases in stretching and grasping motion performances and movement speeds in both groups, which was consistent with the result of this study. Using an upper limb rehabilitation program based on VR for patients with chronic stroke, Perez-Marcos [24] found improvement of upper limb function with increase in shoulder joint AROM and Fugl-Meyer Assessment scores and showed that it was also effective in the follow up period, which was consistent with the result of this study. The result of improvement in the patient's sense of purpose by combining various movements used in actual daily life, and as sense and motor sense signals were transmitted to the subject's hands continuously, this sensory stimulation assisted in making the motion similar to the real one.

In the case of the upper extremity function, the SMVR group showed greater improvement than the VR group. This finding was consistent with that of Mouawad et al. [30] who reported that as a result of a game application, such as tennis, golf, bowling, and baseball to VR, the upper limb function greatly increased, and the group that did not have VR training did not show significant improvements in upper limb function in patients with stroke. Among the subitems of the Jebsen-Taylor hand function test, Merians et al. [31] reported significant improvements in items such as the promotion of writing, card flipping, and pretending to eat, by applying the training of touching the button by the paralyzed upper limb and playing the piano through VR in patients with stroke. In this study, peripheral sensory stimulation was implemented as an intervention, which caused a change in muscle tone, and accordingly, voluntary movement became possible, which affected the promotion of upper limb function.

The present study has a number of limitations. This study included only patients who satisfied the inclusion criteria; thus, it is difficult to generalize the interpretation of our data to the entire stroke population. Because it was not feasible to entirely manipulate the ADLs of the participants and concentration assessment was not performed using various test 
tools, there is a possibility that other factors that cannot be entirely excluded may have influenced the patients. Further studies are warranted in order to examine other pertinent topics, such as training methods of dual tasks and differences in effectiveness based on difficulty levels of the task.

\section{Conflict of Interest}

The authors declared no potential conflicts of interest with respect to the authorship and/or publication of this article.

\section{References}

1. Benjamin EJ, Blaha MJ, Chiuve SE, Cushman M, Das SR, Deo $\mathrm{R}$, et al. Heart disease and stroke statistics-2017 update: a report from the American Heart Association. Circulation 2017;135: e146-603.

2. Writing Group Members, Mozaffarian D, Benjamin EJ, Go AS, Arnett DK, Blaha MJ, et al. Heart disease and stroke statistics-2016 update: a report from the American Heart Association. Circulation 2016;133:e38-360.

3. Jeong TG, Park JS, Choi JD, Lee JY, Kim JS. The effects of sensorimotor training on balance and muscle activation during gait in older adults. J Korean Soc Phys Ther 2011;23:29-36.

4. Park JH, Chung Y. The effects of providing visual feedback and auditory stimulation using a robotic device on balance and gait abilities in persons with stroke: a pilot study. Phys Ther Rehabil Sci 2016;5:125-31.

5. Kang YJ, Park HK, Kim HJ, Lim T, Ku J, Cho S, et al. Upper extremity rehabilitation of stroke: facilitation of corticospinal excitability using virtual mirror paradigm. J Neuroeng Rehabil 2012;9:71.

6. Schaechter JD, van Oers CA, Groisser BN, Salles SS, Vangel MG, Moore CI, et al. Increase in sensorimotor cortex response to somatosensory stimulation over subacute poststroke period correlates with motor recovery in hemiparetic patients. Neurorehabil Neural Repair 2012;26:325-34.

7. de Diego C, Puig S, Navarro X. A sensorimotor stimulation program for rehabilitation of chronic stroke patients. Restor Neurol Neurosci 2013;31:361-71.

8. Lohse KR, Hilderman CG, Cheung KL, Tatla S, Van der Loos HF. Virtual reality therapy for adults post-stroke: a systematic review and meta-analysis exploring virtual environments and commercial games in therapy. PLoS One 2014;9:e93318.

9. Kiper P, Baba A, Agostini M, Turolla A. Proprioceptive based training for stroke recovery. Proposal of new treatment modality for rehabilitation of upper limb in neurological diseases. Arch Physiother 2015;5:6.

10. Saito K, Yamaguchi T, Yoshida N, Tanabe S, Kondo K, Sugawara K. Combined effect of motor imagery and peripheral nerve electrical stimulation on the motor cortex. Exp Brain Res 2013;227:333-42.

11. Kwakkel G, van Peppen R, Wagenaar RC, Wood Dauphinee S, Richards C, Ashburn A, et al. Effects of augmented exercise ther- apy time after stroke: a meta-analysis. Stroke 2004;35:2529-39.

12. Sousa Nanji L, Torres Cardoso A, Costa J, Vaz-Carneiro A. Analysis of the Cochrane review: interventions for improving upper limb function after stroke. Cochrane Database Syst Rev. 2014,11:CD010820. Acta Med Port 2015;28:551-3.

13. Maggio MG, Latella D, Maresca G, Sciarrone F, Manuli A, Naro A, et al. Virtual reality and cognitive rehabilitation in people with stroke: an overview. J Neurosci Nurs 2019;51:101-5.

14. Tieri G, Morone G, Paolucci S, Iosa M. Virtual reality in cognitive and motor rehabilitation: facts, fiction and fallacies. Expert Rev Med Devices 2018;15:107-17.

15. Flynn S, Palma P, Bender A. Feasibility of using the Sony PlayStation 2 gaming platform for an individual poststroke: a case report. J Neurol Phys Ther 2007;31:180-9.

16. Song $\mathrm{CH}$, Seo SM, Lee KJ, Lee YW. Video Game-Based Exercise for upper-extremity function, strentgth, visual perception of stroke patients. J Spec Educ Rehabil Sci 2011;50:155-80.

17. Kober SE, Wood G, Hofer D, Kreuzig W, Kiefer M, Neuper C. Virtual reality in neurologic rehabilitation of spatial disorientation. J Neuroeng Rehabil 2013;10:17.

18. Smania N, Montagnana B, Faccioli S, Fiaschi A, Aglioti SM. Rehabilitation of somatic sensation and related deficit of motor control in patients with pure sensory stroke. Arch Phys Med Rehabil 2003;84:1692-702.

19. Kolber MJ, Hanney WJ. The reliability and concurrent validity of shoulder mobility measurements using a digital inclinometer and goniometer: a technical report. Int J Sports Phys Ther 2012;7:306-13.

20. Boggio PS, Castro LO, Savagim EA, Braite R, Cruz VC, Rocha $R R$, et al. Enhancement of non-dominant hand motor function by anodal transcranial direct current stimulation. Neurosci Lett 2006;404:232-6.

21. Artilheiro MC, Fávero FM, Caromano FA, Oliveira ASB, Carvas N Junior, Voos MC, et al. Reliability, validity and description of timed performance of the Jebsen-Taylor Test in patients with muscular dystrophies. Braz J Phys Ther 2018;22:190-7.

22. Miyamoto S, Kondo T, Suzukamo Y, Michimata A, Izumi S. Reliability and validity of the Manual Function Test in patients with stroke. Am J Phys Med Rehabil 2009;88:247-55.

23. Raine S, Meadows L, Lynch-Ellerington M. Bobath concep: theory and clinical practice in neurological rehabilitation. Oxford: Wiley-Blackwell; 2009.

24. Perez-Marcos D, Chevalley O, Schmidlin T, Garipelli G, Serino $\mathrm{A}$, Vuadens $\mathrm{P}$, et al. Increasing upper limb training intensity in chronic stroke using embodied virtual reality: a pilot study. J Neuroeng Rehabil 2017;14:119.

25. Kim JH, Lee BH. Mirror therapy combined with biofeedback functional electrical stimulation for motor recovery of upper extremities after stroke: a pilot randomized controlled trial. Occup Ther Int 2015;22:51-60.

26. Powell J, Pandyan AD, Granat M, Cameron M, Stott DJ. Electrical stimulation of wrist extensors in poststroke hemiplegia. Stroke 1999;30:1384-9.

27. Yun GJ, Chun MH, Park JY, Kim BR. The synergic effects of mirror therapy and neuromuscular electrical stimulation for hand function in stroke patients. Ann Rehabil Med 2011;35:316-21.

28. Lee D, Lee M, Lee K, Song C. Asymmetric training using virtual reality reflection equipment and the enhancement of upper limb 
function in stroke patients: a randomized controlled trial. J Stroke Cerebrovasc Dis 2014;23:1319-26.

29. Subramanian SK, Levin MF. Viewing medium affects arm motor performance in 3D virtual environments. J Neuroeng Rehabil 2011;8:36

30. Mouawad MR, Doust CG, Max MD, McNulty PA. Wii-based movement therapy to promote improved upper extremity function post-stroke: a pilot study. J Rehabil Med 2011;43:527-33.

31. Merians AS, Poizner H, Boian R, Burdea G, Adamovich S. Sensorimotor training in a virtual reality environment: does it improve functional recovery poststroke? Neurorehabil Neural Repair 2006;20:252-67. 